\title{
Quantitative Service Analysis
}

\author{
Naveen Kulkarni, Deepti Parachuri, and Shashank Trivedi \\ Software Engineering and Technology Labs, Infosys Technologies Ltd, India \\ \{Naveen_Kulkarni, Deepti_Parachuri, Shshank_Trivedi\}@infosys.com
}

\begin{abstract}
Service Orientation has become popular due to dynamic market conditions and changing customer needs. A successful service oriented architecture implementation requires the need for right identification of services from business process models. Service identification is considered to be the main activity in the modeling of service oriented solution, as errors made during service identification flows down through detailed design and implementation of activities.

Though service orientation has been an important milestone in many enterprise transformation initiatives, there hasn't been much work on identification of services. Services have been identified and are used in day to day transactions, but they are limited to exchange of information between partners (two different organizations) or infrastructure related. Functionalities that are widely used across all applications such as security, auditing has been considered for servicification. In some other cases, business processes have been considered as simple orchestrated set of web services with each activity mapping to a single web service.

Adopting any service identification approach for Service Orientation without verification would rather be impractical for the simple reason being that no common notion of service can be established among stakeholders. It is essential to assert if all services identified provide necessary value and exhibit acceptable technical health (flexibility, reuse etc). To be more effective, there is a need for a methodology that can quantitatively measure the candidature of services with respect to business process models. With such automation, a platform can be provided to bootstrap service analysis where stakeholders can continually model and refine services based on predefined criteria.

This tutorial is intended for researchers and industry practitioners who are interested in Service Oriented Architecture and Service Analysis. The tutorial gives a deeper insight on service analysis and service identification methodologies. Though our methodology follows the prescribed top down approach while recognizing the importance of starting with business models for service identification, it stands different as it is based on mathematical model rather than heuristics or questionnaire based. Our method adopts quantitative way of groping set of business activities and measuring the service candidacy of those groups based on well defined principles. It also demonstrates an automated tool for service analysis.

abstract environment.
\end{abstract}

